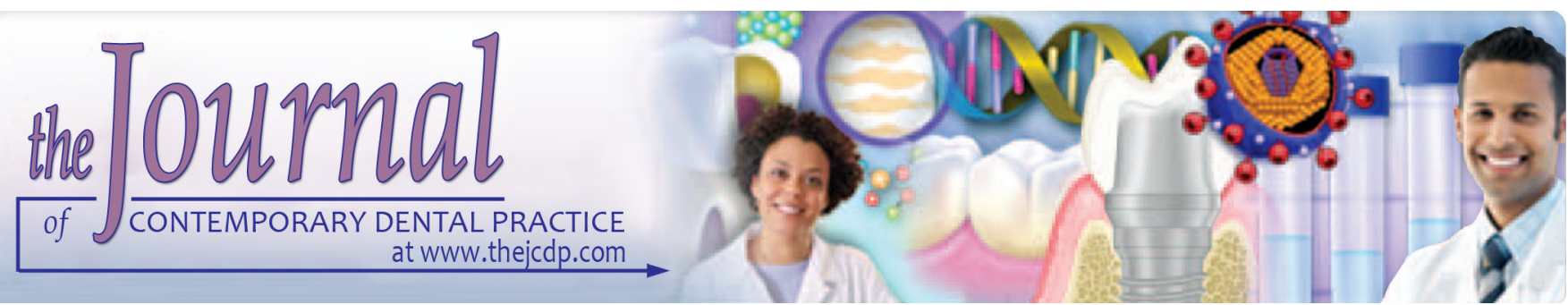

\title{
To Assess and Evaluate the Variation of Mandibular Anatomy Using Cone Beam Computed Tomography Before Planning an Implant Surgery: A Population-based Retrospective Study
}

\begin{abstract}
Aim: The accurate placement of implants in mandible requires consideration for angulations of the bone along with the vertical dimensions. The aim of this present study was to assess the variation of mandibular anatomy using computed tomography (CT) radiography and to evaluate the effect of presence and absence of teeth on the mandibular anatomy before planning an implant surgery.
\end{abstract}

${ }^{1}$ Vikram Kapoor, ${ }^{2}$ Narendra Kumar, ${ }^{3}$ Kirti Dahiya, ${ }^{4}$ Rohan Sikka, ${ }^{5}$ Pallavi Sirana, ${ }^{6}$ Abhinav Singh
Material and methods: The present population-based retrospective study was conducted using 167 digital CT scan images those selected from departmental archives. The samples were sub-divided two groups: group 1included digital CT of edentulous mandible while group 2 included digital CT of edentulous mandible. The axial height, vertical height, and angulations were recorded separately for each group.

Results: The results of the present study showed a gradual increase in mandibular angle in both the groups with a statistically significant difference only in the canine-premolar area. The axial height showed a significant difference at canine and first premolar area and a second premolar and the first molar area. However, available height showed a significant difference in canine and first premolar area and distal to the second molar area. When both the sides were compared, no statistically significant difference was observed between right and left sides.

Conclusion: It was concluded that due to the variability of the mandibular anatomy and because of the effects of various imperative factors, 3D imaging like CT scans should be recommended for safer and secure presurgical planning.

\footnotetext{
1-3,5Department of Prosthodontics, Institute of Dental Studies and Technologies, Modinagar, Uttar Pradesh, India

${ }^{4}$ Department of Prosthodontics, PDM Dental College and Research Institute, Bahadurgarh, Haryana, India

${ }^{6}$ Department of Orthodontics, DJ Dental College, Modinagar, Uttar Pradesh, India

Corresponding Author: Vikram Kapoor, Department of Prosthodontics, Institute of Dental Studies and Technologies, Modinagar, Uttar Pradesh, India Phone: +918882981272, e-mail:kaps5455@gmail.com
}

Clinical significance: In surgical osteotomies and implant placement especially in post-extraction sockets, two-dimensional (2D) image of panoramic radiographs should not be considered that reliable as these three-dimensional (3D) imaging radiographs. Therefore $\mathrm{CT}$ scans of various angulations and sections must be considered by the clinicians to rationally study the mandibular anatomy and their risk associated areas.

Keywords: Angulation, Axial height, Cone beam computed tomography (CBCT), Mandible, Population.

How to cite this article: Kapoor V, Kumar N, Dahiya K, Sikka R, Sirana P, Singh A. To Assess and Evaluate the Variation of Mandibular Anatomy Using Cone Beam Computed Tomography Before Planning an Implant Surgery: A Population-based Retrospective Study. J Contemp Dent Pract 2018;19(11): 1381-1386.

Source of support: Nil

Conflict of interest: None

\section{INTRODUCTION}

With the invention of dental implants, the oral field rehabilitation of missing teeth has been revolutionized. Dental implants and 3D imaging techniques have revolutionized the field oral rehabilitation of missing teeth. A successful dental implants therapy largely depends on the thorough evaluation of the jaw bones related to its quantity as well as quality. ${ }^{1,2}$ For the best placements of implants without any surgical complication, complete knowledge of the bone topography including the defects must be considered. Therefore, the role of radiography becomes imperative here. In earlier times, conventional techniques like panoramic radiographs became popular and were considered as the most commonly used imaging modality for implant planning. ${ }^{3,4}$ However with the advent of sectional imaging techniques like CT scans, the world of diagnosis and radiography was revolutionized for rehabilitation procedures. ${ }^{5}$ The American Academy of Oral and Maxillofacial Radiology (AAOMR) has recently recommended CBCT as the best option in the diagnostic 
field. ${ }^{6}$ It is well known that mandibular teeth naturally have a slight lingual inclination to both the mandibular body and maxillary arch. ${ }^{7}$ Thus, placement of implants in mandible requires consideration for angulation of the bone along with the vertical dimensions. Also, it's been reported in the literature that the anatomy of the mandible in presence and absence of teeth vary because of the bone remodeling. This variation causes decreased available vertical height of the alveolar ridge, its width, and even the angle which may further complicate the implant placement. ${ }^{8}$ Literature has also well evidenced by the location of anatomical structures, and the amount of residual alveolar bone available for osteotomy vary among different populations. ${ }^{9,10}$ Thus, the aim of this present study was to assess the variation of mandibular anatomy using CT radiography in institutionalized population and to evaluate the effect of presence and absence of teeth on the mandibular anatomy before planning an implant surgery.

\section{MATERIAL AND METHODS}

The present population-based retrospective study was conducted in the Department of Prosthodontics Institute of Dental Studies and Technology, Modinagar. Ethical clearance for the present study was taken from the institutional ethical committee. All patients had provided us signed informed consent as an agreement to participate in the study. A total of 167 digital CT scan images were selected from departmental archives of last 3 years. The ages of the selected sample ranged from 20 to 65 years with 98 males and 69 females belonging to the local population of the surrounding area of the institute. According to the inclusion criteria both dentulous as well as edentulous good quality digital CT images without any artifacts of the mandible were included in the study. We had divided the study sample into two major groups: group 1: included digital CT of edentulous mandible and group 2: included digital CT of edentulous mandible. The CT scans with pathologies of the mandible, trauma cases or with severe atrophy of the alveolar ridges in edentulous cases were excluded from the study. Once the desired sample size is obtained after the inclusion and exclusion criteria, all the digital CTs were prepared with a $0^{\circ}$ gantry tilt using the automated calibrated software. All the observations were performed by a single experienced implantologist to minimize observer bias. Reference points in the study were similar to those taken by Sammartino et al. in their study ${ }^{10}$ Group 1 samples included dentate mandibles wherein following three reference areas were recorded: (1) area between two mandibular central incisors (symphysis), (2) area between canine and first premolar of both right and left side, (3) areas between the second premolar and the first molar on the right and left and area distal to 2nd molar on both right and left side. Similarly, for group 2 (which included edentulous mandibular samples), the following areas were recorded; a slice between the apophysis genii which was considered as a median reference point and rest all the measurements were calculated as a mean distance from this median reference in the CT. Thus, for all the above reference points mentioned in both the groups; axial height, vertical height and angulations were recorded separately. Also, specifically in inter mental foramen area; the axial height and the available distance to place the implant were compared (Figs 1 to 3 ).

\section{STATISTICAL ANALYSIS AND RESULTS}

All obtained observations were tabulated and sent for statistical analysis. Statistical analysis was completed using the statistical software 'Statistical Package for the Social Sciences' version 21 (IBM Inc., Armonk, New York, USA). To obtain the $\mathrm{p}$ values t-test was applied. A p-value $<0.005$ were considered as statistically significant. The present study comprised of a total sample size of 167 mandibular digital computed tomographies of the patients. The characteristics of the sample are tabulated in Table 1. The results of the present study shows that both in group1 and group 2 subjects; gradual increase in mandibular angle both anteroposterior and buccolingual direction with mandibular central incisor area showing $8.85 \pm 2.4$ in group $1,10.02 \pm 5.4$ in group 2 and $14.98 \pm$ 1.13 and $15.27 \pm 3.24$ in 2 nd molar area in groups 1 and 2 respectively (Table 2 ). Though we observed increased angulation values in group 2 in comparison to group 1 , this difference was statistically significant only in the caninepremolar area $(p<0.005)$. The mean values of axial heights and available height for implant placement observed in both the groups are tabulated in Table 3 . In relation to axial height, a significant difference was observed between both the groups at canine and first premolar area and a second premolar and the first molar area. On further comparison of mean values of available height, a significant difference was observed between the groups at canine and first premolar area and distal to the 2nd molar area. When both the sides were compared no statistically significant difference was observed between right and left sides.

\section{DISCUSSION}

In this era of advancements in dentistry, it has become more challenging to replace a missing tooth then extracting and restoring the same. Implants have thus become the treatment of choice by most of the practitioners as well as patients themselves. Thus, considering this, 


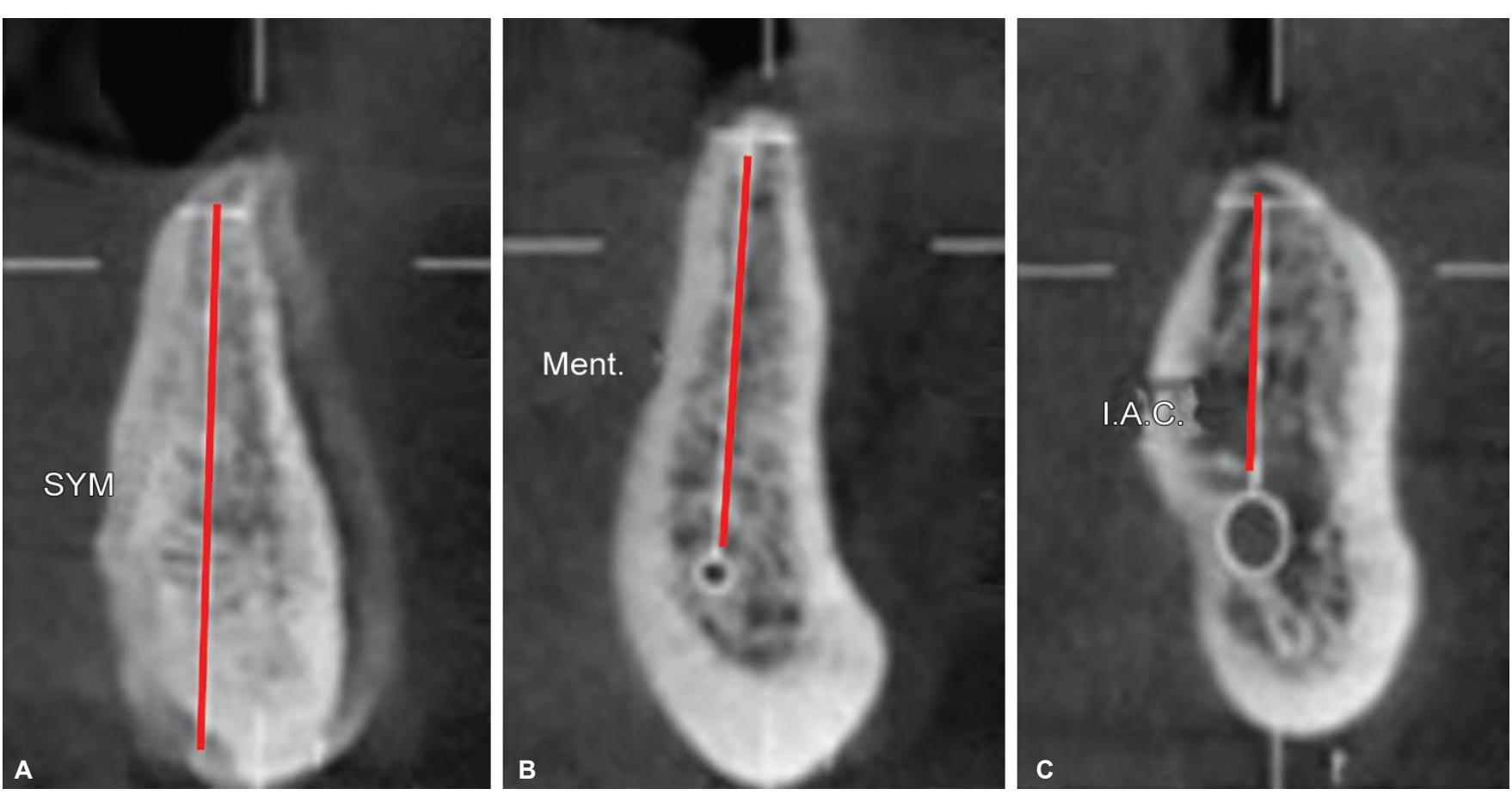

Figs $1 \mathrm{~A}$ to $\mathrm{C}$ : Sample estimation of suitable implant length on CBCT slices at different region of dentulous jaw (A) The distance between the mid crestal point of the ridge and the lowest point along the axis of the crest (at symphysial region); (B) the distance from the mid crestal point to the mental foramen opening; $(C)$ the distance from the mid crestal point to the mental foramen opening (estimated at the course of Inferior alveolar canal)

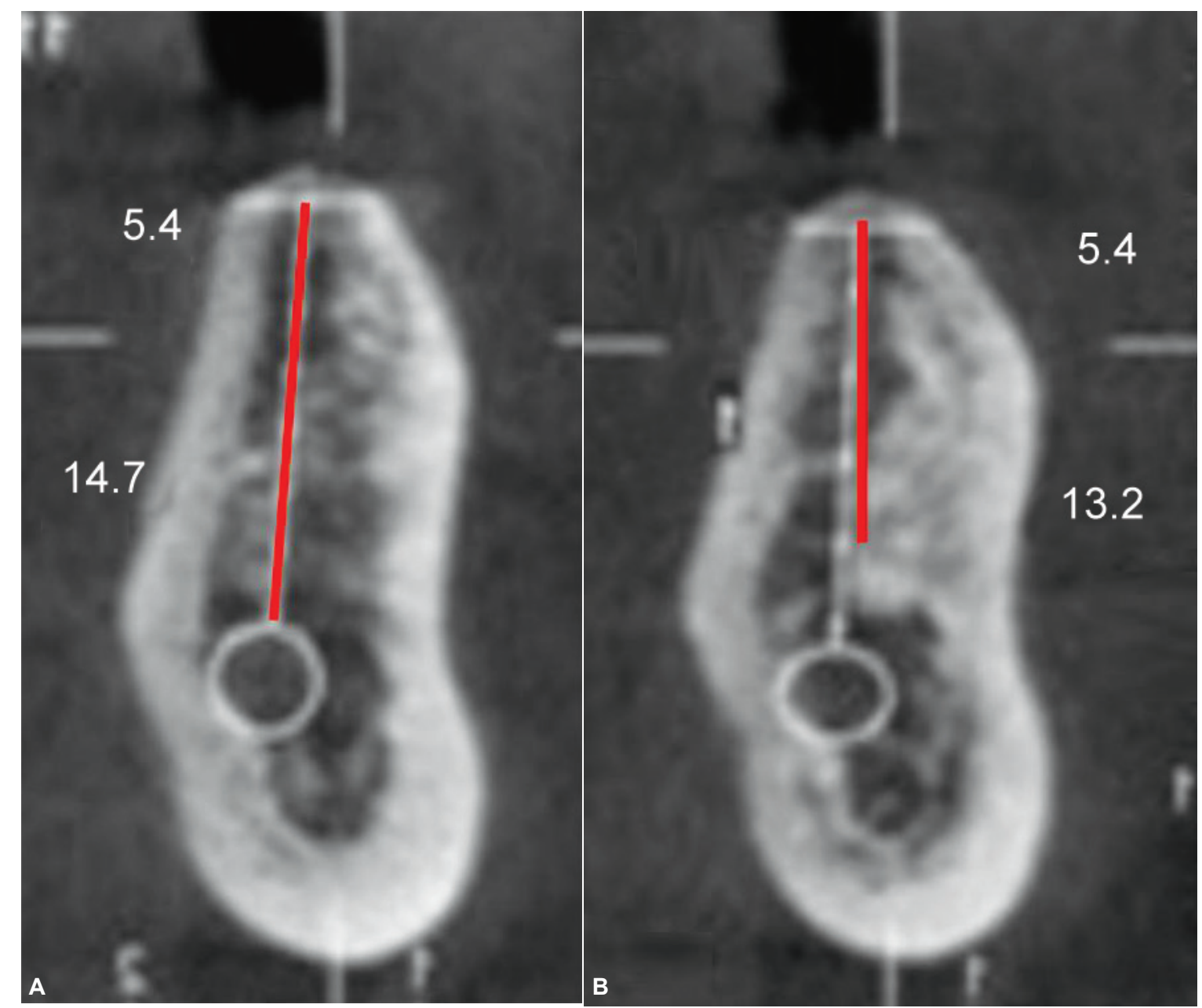

Figs 2A and B: (A) The distance between the mid crestal point of the ridge and mental foramen along the axis of bony crest; (B) The approximate available space for placement of the implant (1.5 $\mathrm{mm}$ short to the mental foramen) 


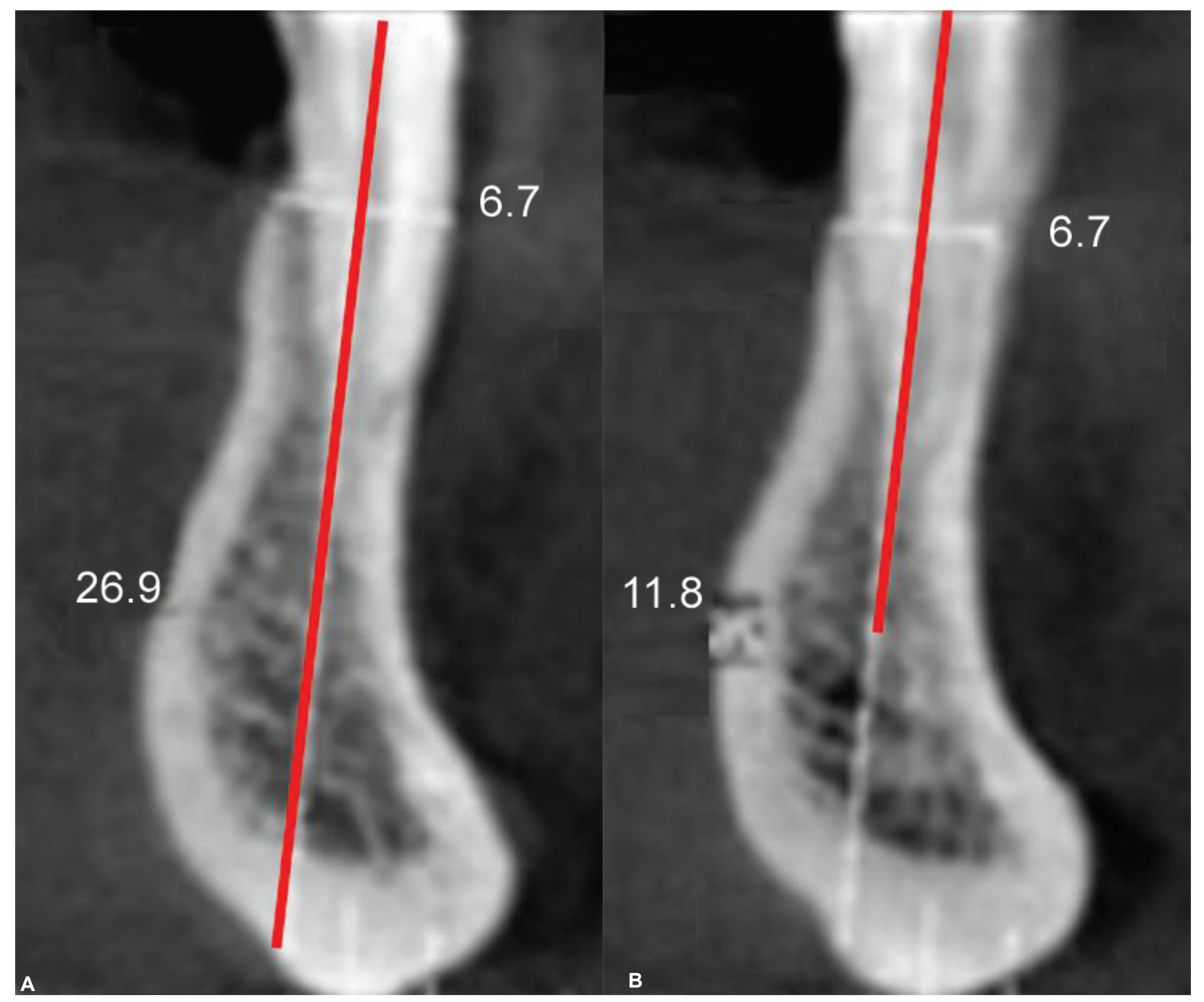

Figs $3 A$ to and B: (A) The distance from the centre of the dentulous ridge to the base through inferior alveolar nerve; (B) The approximate distance available for implant placement

supportive aids like appropriate diagnostic tools like radiography, proper treatment planning and choice of implants according to the bone availability have become

Table 1: Shows study sample characteristics

\begin{tabular}{ll}
\hline Study characteristics & Mean values \\
\hline Sample size & \\
Total & 167 \\
Group 1 & 85 \\
Group 2 & 82 \\
\hline Gender & \\
Males & 98 \\
Females & 69 \\
Age & 42.85 years \\
\hline
\end{tabular}

an important and challenging step in the success of an implant in clinical practice. ${ }^{11,12}$ Radiographic examination during diagnosis and treatment is a foremost and major step, as it not only provides us with the information about the location of anatomical structures but also helps in the estimation of the amount of bone available for placement of the implant and identifying any intrabony pathologies which may affect the prognosis of the treatment. CT scans are three-dimensional radiographs which have been evolved as a great help in diagnosis and treatment planning in clinical dentistry. Currently available nextgeneration CT scanners can produce self-formatted images with a slice width of $0.25 \mathrm{~mm}$ pixel size and an

Table 2: Shows mean values of angulation recorded from different reference points in both the study groups

\begin{tabular}{lll}
\hline Reference Areas & Group1 & Group 2 \\
\hline Between mandibular central incisor & $8.85 \pm 2.4$ & $10.02 \pm 5.4$ \\
Between Canine and first premolar & $7.36 \pm 0.45$ & $10.98 \pm 0.25$ \\
Between second premolar and the first molar & $10.14 \pm 1.52$ & $11.58 \pm 3.62$ \\
Distal to 2nd molar & $14.98 \pm 1.13$ & $15.27 \pm 3.24$ \\
\hline
\end{tabular}


Table 3: Shows mean axial height and available height for implant placement. At all the reference points in both the groups

\begin{tabular}{|c|c|c|c|c|}
\hline & \multicolumn{2}{|c|}{ Axial height } & \multicolumn{2}{|c|}{ Available height for implant placement } \\
\hline & Group 1 & Group 2 & Group 1 & Group 2 \\
\hline Between mandibular central incisor & $29.42 \pm 2.5$ & $27.48 \pm 3.6$ & $25.84 \pm 4.7$ & $24.32 \pm 4.6$ \\
\hline Between Canine and first premolar & $26.25 \pm 2.4$ & $22.23 \pm 4.50$ & $22.21 \pm 3.4$ & $17.46 \pm 4.3$ \\
\hline $\begin{array}{l}\text { Between second premolar and the } \\
\text { first molar (above mandibular canal) }\end{array}$ & $17.38 \pm 3.2$ & $12.51 \pm 4.6$ & $14.12 \pm 2.6$ & $11.02 \pm 21$ \\
\hline
\end{tabular}

in-plane resolution of one pixel by the scan spacing 0.5 to $1.5 \mathrm{~mm}$. These programming ad calibrations produce a geometric resolution that similar to planar imaging. Thus, this technique allows sectional imaging of the jaws, as per requirement and interest of the observer which can be either a whole view or a restricted specific area view. ${ }^{13}$ It has been said that $\mathrm{CT}$ scan should be performed routinely before planning an implant, especially in the infra-orbital region because of its good image quality and better reproducibility, and less radiation exposure. Therefore, in our study, we used digital CT scans to outline the mandibular morphology and estimate the associated risk areas for placing implants. Following strict and specific inclusion and exclusion criteria, we used digital CT scans of the selected subjects from departmental archives. Being a population-based study, we focused on the north Indian population. As already shown that available amount of bone is not just influenced by the anatomic structures, but also factors such as gender and race, therefore we tried to include a wide age range and considered gender status as well in our study. ${ }^{14}$ It was observed that age and bone height showed an inverse relation, i.e., greater the age, lesser was the available bone height for implant placement. Gender difference did not prove to be much related to available bone height in our study. In their review, Lingam et al. also mentioned a similar pattern which was in accordance with our study. ${ }^{15}$ Considering the importance of mandibular teeth inclination with the mandibular base and with a base of the skull, we focused on calculating the mean values of angulations mandible in both the presence and absence of teeth.

From our results, we showed a gradual increase in mandibular angle both anteroposterior and buccolingual in both the study groups. It was also observed that the mean angulation of the mandible in group 2, which had edentulous mandibles showed greater angulation when compared with group 1 which included dentate group, but the difference was statistically significant only in canine premolar $(p<0.005)$. Gender and age did not show any significant relation to angulation in this population. In accordance with our study similar results were reported by Sammartino et al., ${ }^{10}$ who also reported the statistically significant difference in the second premolar-first molar area. They reported that these results set a restriction on the implant positioning according to an ideal prosthetic axis thus limiting the use of angled abutments which in turn increases stress and strain on the bone. On contrary, Sethi et al. reported that angles did not significantly influence the overall implant survival rate ${ }^{16}$ Considering the axial height and available bone height it was observed that edentulous cases have decreased axial height in comparison with dentulous cases. This difference was most significant in both canines-first premolar area and second premolar-first molar areas. These results thus focus on the fact that, middle areas of the mandible are more prone to such height changes in comparison to anterior and posterior areas. ${ }^{17,18}$ Our results are supported by the work of other authors as well who documented that marked height differences are seen in the presence and absence of teeth. ${ }^{8,9}$ Further on a comparison of mean values of available height for implant placement, a significant difference was noticed at canine-first premolar area and distal to the second molar region. However, no statistically significant difference was noticed between right and left sides. This study design has its limitations like lesser sample size to generalize the results of a particular population. Secondly, comparisons with other commonly used radiographic techniques should be done to lay more emphasis on the usage of CT scans for implant placement by the clinicians. ${ }^{19}$ The clinical significance of this study lies on the advantage and importance of considering mandibular angulation during treatment planning along with the axial height of the available bone. Literature shows the failure of implants due to high risk of lingual plate perforations even in cases with adequate alveolar height. ${ }^{20-23}$ Thus, we stress on the fact that during implant placement especially in postextraction sockets, 2D image of panoramic radiographs should not be considered that reliable as these $3 \mathrm{D}$ imaging radiographs. Thus, to achieve best results, CT scans should be considered as best choice by the clinicians to study the mandibular anatomy and their risk associated areas.

\section{CONCLUSION}

With the advancements in implant imaging techniques, conventional radiography has been obsolete. Some panoramic radiography is still in use only because of their low costs. However, it is merely difficult to precisely locate 
the vital structures by using them. With the introduction of computed tomographies, qualitative and quantitative bony assessments with $3 \mathrm{D}$ reconstructions have become possible. Our study results showed that due to the variability of the mandibular anatomy and because of other factors, 3D imaging like CT scans should be recommended for safer pre-surgical planning. This helps in preventing the unwanted complications like perforations, especially due to angulation of teeth in mandible during implant placement surgical procedures. Our study results can be treated as suggestive for formulating accurate treatment planning particularly for length and diameter of the implant. However, we foresee other large-scale studies to be conducted that could further establish certain authentic guidelines in this prospect.

\section{CLINICAL SIGNIFICANCE}

Three-dimensional imaging offers rapid data compilation with minimum radiation exposure compared to those with panoramic ones. We stress on the fact that during implant placement especially in post-extraction sockets, 2D image of panoramic radiographs should not be considered that reliable as these $3 \mathrm{D}$ imaging radiographs. Thus, to achieve the most excellent results, CT scans should be considered as best choice by the clinicians to study the mandibular anatomy and their risk associated areas.

\section{REFERENCES}

1. Mozzo P, Procacci C, Tacconi A, Tinazzi Martini P, Andreis IB. A new volumetric CT machine for dental imaging based on the cone-beam technique: preliminary results. Eur Radiol 1998;8(9):1558-1564.

2. Tyndall DA, Price JB, Tetradis S, Ganz SD, Hildebolt C, Scarfe WC. Position statement of the American Academy of Oral and Maxillofacial Radiology on selection criteria for the use of radiology in dental implantology with emphasis on cone beam computed tomography. Oral Surg Oral Med Oral Pathol Oral Radiol 2012;113(6):817-826.

3. Harris D, Buser D, Dula K, Grondahl K, Haris D, Jacobs R. Guidelines for the use of diagnostic imaging in implant dentistry: A consensus workshop organized by the European Association for Osseo-integration in Trinity College Dublin. Clin Oral Implants Res 2002;13(5):566-570.

4. Ishanti R, Rao G. Implant imaging. Int J Innov Res Dev 2013;2:285-289.

5. Juodzbalys G, Kubilius M. Clinical and radiological classification of the jawbone anatomy in endosseous dental implant treatment. J Oral Maxillofac Res 2013;4:e2.

6. Tyndall DA, Price JB, Tetradis S, Ganz SD, Hildebolt C, Scarfe WC. Position statement of the American Academy of Oral and Maxillofacial Radiology on selection criteria for the use of radiology in dental implantology with emphasis on cone beam computed tomography. Oral Surg Oral Med Oral Pathol Oral Radiol 2012;113(6):817-826.
7. Misch K, Wang HL. Implant surgery complications: aetiology and treatment. Impl Dent 2008;17(2):159-168.

8. Covani U, Ricci M, Bozzolo G, Mangano F, Zini A, Barone A. Analysis of the pattern of the alveolar ridge remodelling following single tooth extraction. Clin Oral Imp Res 2011; 22(8):820-825.

9. Van der Weijden F, Dell'Acqua F, Slot DE. Alveolar bone dimensional changes of post-extraction sockets in humans: a systematic review. J Clin Periodontol 2009;36(12):1048-1058.

10. Sammartino G, Prados-Frutos JC, Riccitiello F. The Relevance of the Use of Radiographic Planning in Order to Avoid Complications in Mandibular Implantology: A Retrospective Study. Bio Med Res Int 2016;7:1-7.

11. Misch CE. Density of bone: effect on treatment plans, surgical approach, healing, and progressive bone loading. Int J Oral Implantol 1990;6(2):23-31.

12. Quirynen M, Van Assche N, Botticelli D, Berglundh T. How does the timing of implant placement to extraction affect outcome? Int J Oral Maxillofac Implants 2007;22(7):203-223.

13. Schwarz MS, Rothman SL, Rhodes ML, Chafetz N. Computed tomography: Part I. Preoperative assessment of the mandible for endosseous implant surgery. Int J Oral Maxillofac Implants 1987;2(3):137-141.

14. Peltola JS, Mattila M. Cross-sectional tomograms obtained with four panoramic radiographic units in the assessment of implant site measurements. Dentomaxillofac Radiol 2004;33(5):295-300.

15. Lingam AS, Reddy L, Nimma V, Pradeep K. “Dental implant radiology" - Emerging concepts in planning implants. J Orofac Sci 2013;5(2):88-94.

16. Sethi A, Kaus T, Sochor P, Axmann-Krcmar D, Chanavaz M. Evolution of the concept of angulated abutments in implant dentistry: 14-year clinical data. Imp Dent 2002;11(1):41-51.

17. Athota A, Gandhi Babu DB, Nagalaxmi V, RaghojiS, Waghray S, Reddy CR. A comparative study of digital radiography, panoramic radiography, and computed tomography in dental implant procedures. J Indian Acad Oral Med Radiol 2017;29(2):106-110.

18. Tang Z, Liu X, Chen K. Comparison of digital panoramic radiography versus cone beam computerized tomography for measuring alveolar bone. Head \& face medicine. 2017 Dec;13(1):1-7.

19. Shruthi M, Sangeetha R, Singh AK, Kini R, Naik V. To implant or not to implant?: The role of imaging. J Orofac Res 2013:3:210-217.

20. Gupta S , Patil N , Solanki J , Singh R , Laller S. Oral Implant Imaging: A Review. Malays J Med Sci 2015;22(3):7-17.

21. Alnahwi M, Alqarni A, Alqahtani R, Magnas BB , Alshahrani FN. A survey on radiographic prescription practices in dental implant assessment. J Applied Dent Med Sci 2017;3:148-56.

22. Sahota J, Bhatia A, Gupta M, Singh V, Soni J, Soni R. Reliability of Orthopantomography and Conebeam Computed Tomography in Presurgical Implant Planning: A Clinical Study. J Contemp Dent Pract 2017;18(8):1-5.

23. Garlapati K, Babu DBG, Chaitanya NCSK, Guduru H, Rembers A, Soni P. Evaluation of Preference and Purpose of Utilisation of Cone Beam Computed Tomography (CBCT) Compared to Orthopantomogram (OPG) by Dental Practitioners-A Cross-Sectional Study. Polish J Radiol 2017;82:248-251. 